\title{
Conceptual Analysis of Electron Transpiration Cooling for the Leading Edges of Hypersonic Vehicles
}

\author{
Hicham Alkandry, ${ }^{*}$ Kyle M. Hanquist,$^{\dagger}$ and Iain D. Boyd ${ }^{\ddagger}$ \\ Department of Aerospace Engineering, University of Michigan, Ann Arbor, MI
}

\begin{abstract}
Recent progress is presented in an ongoing effort to perform a conceptual analysis of possible electron transpiration cooling using thermo-electric materials at the leading edges of hypersonic vehicles. The implementation of a new boundary condition in the CFD code LeMANS to model the thermionic emission of electrons from the leading edges of hypersonic vehicles is described. A parametric study is performed to understand the effects of the material work function, the freestream velocity, and the leading edge geometry on this cooling effect. The numerical results reveal that lower material work functions, higher freestream velocities, and smaller leading edges can increase the cooling effect due to larger emission current densities. The numerical results also show that the electric field produced by the electron emission may not have a significant effect on the predicted properties. Future work recommendations are provided that may improve the physical accuracy of the modeling capabilities used in this study.
\end{abstract}

\section{Nomenclature}

$A_{R} \quad$ Richardson constant

$C_{s} \quad$ Charge of species $s$

$D_{s} \quad$ Diffusion coefficient of species $s$

E $\quad$ Electric field

$\mathbf{j}_{e} \quad$ Electric current density

$J_{e} \quad$ Emission current density

$k_{b} \quad$ Boltzmann constant, $1.38 \times 10^{-23} \mathrm{~J} / \mathrm{K}$

$\dot{m} \quad$ Mass blowing rate

$M_{s} \quad$ Molar mass of species $s$

$N_{A} \quad$ Avogadro constant, $6.02 \times 10^{23} \mathrm{~mol}^{-1}$

$n_{s} \quad$ Number density of species $s$

NS Number of species

$p \quad$ Pressure

$Q_{e} \quad$ Elementary charge, $1.60 \times 10^{-19} \mathrm{C}$

$q_{w} \quad$ Heat transfer to the vehicle

$R_{n} \quad$ Nose radius

$R_{u} \quad$ Universal gas constant, $8.31 \mathrm{~J} / \mathrm{K} / \mathrm{mol}$

$T \quad$ Temperature

U Velocity

$\dot{w}_{s} \quad$ Production rate of species $s$

$Y_{s} \quad$ Mass fraction of species $s$

$\epsilon \quad$ Material emissivity

$\epsilon_{0} \quad$ Vacuum permittivity, $8.85 \times 10^{-12} \mathrm{~F} / \mathrm{m}$

$\phi \quad$ Electric potential

$\Phi \quad$ Material work function

* Research Fellow, Member AIAA.

† Graduate Student Research Assistant, Student Member AIAA.

$\ddagger$ James E. Knott Professor of Engineering, Fellow AIAA. 


$\begin{array}{ll}\rho & \text { Density } \\ \sigma & \text { Electric conductivity } \\ \sigma_{s b} & \text { Stefan-Boltzmann constant, } 5.67 \times 10^{-8} \mathrm{~W} / \mathrm{m}^{2} / \mathrm{K}^{4} \\ & \\ \text { subscripts } & \\ \infty & \text { Freestream } \\ w & \text { Wall }\end{array}$

\section{Introduction}

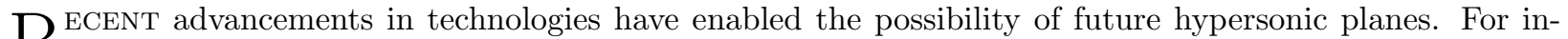
R stance, a joint program called Falcon was initiated by the Defense Advanced Research Projects Agency (DARPA) and the United States Air Force in 2003 to advance the technology required for sustained hypersonic flight. ${ }^{1}$ This program produced two hypersonic flight tests called Hypersonic Technology Vehicle 2 (HTV-2) in 2010 and 2011. Such hypersonic vehicles require the use of sharp leading edges in order to increase the aerodynamic lift-to-drag ratio and maximize range. However, the aerodynamic performance gains offered by sharp leading edges for hypersonic vehicles come at the cost of very high, localized heating rates. A theoretical analysis first performed by Fay and Riddell ${ }^{2}$ reveals that the stagnation point heat transfer is inversely proportional to the radius of the vehicle leading edge as shown in Equation 1,

$$
q_{w} \propto \sqrt{\frac{\rho_{\infty}}{R_{n}}} U_{\infty}^{3}
$$

where $\rho_{\infty}$ and $U_{\infty}$ are the freestream mass density and velocity, respectively, and $R_{n}$ is the radius of the leading edge. This increase in the aerodynamic heating causes the temperature at the leading edge to reach well over $2000 \mathrm{~K}$ for hypersonic flight. As a result, hypersonic vehicles use a thermal protection system (TPS) to insulate the vehicle from these extreme temperatures. Current TPS materials, however, may be inappropriate for hypersonic planes because they either cannot sustain the large heating rates or they are ablative and not reusable.

One approach to solve this issue of high localized heating rates is to use ultra high temperature composite (UHTC) materials at the leading edges of the hypersonic vehicle. This approach was used on the NASA X-43 experimental hypersonic aircraft, which reached Mach 10 during its final flight in $2004 .{ }^{3}$ UHTC materials, however, have physical limitations such as heavy weight and weak fracture toughness that may prevent their use in some future hypersonic vehicles. ${ }^{4}$ Another approach that has been proposed involves the use of thermo-electric materials at the leading edges of hypersonic vehicles. These materials can generate a current of electrons when exposed to high temperatures that may lead to a transpiration cooling effect of the vehicle surface. This phenomenon is known as thermionic electron emission and occurs when the thermal energy given to the electrons (by aerodynamic heating in this case) is greater than the binding potential of the surface material.

Assuming that such thermo-electric materials exist with thermal and physical properties that can withstand the conditions of hypersonic flight, an initial conceptual analysis of the aerothermal environment experienced by the hypersonic vehicle using computational fluid dynamics (CFD) is the most appropriate starting point since experimental studies of such systems would be both expensive and technically challenging. Therefore, this paper aims to present a conceptual analysis of the aerothermodynamic characteristics of hypersonic vehicles with thermo-electric materials using CFD. The paper is presented as follows. First, a description of the numerical method and test case conditions are outlined. Then, numerical results are presented that highlight the potential cooling effect provided by thermo-electric TPS materials. Next, a parametric study is performed to understand the influence of material work function, freestream velocity, and leading edge geometry on this cooling phenomenon. The effects of the electric field generated by the thermionic electron emission are then estimated and discussed. Finally, the paper presents some conclusions drawn from this study and outlines future work recommendations. 


\section{Numerical Approach}

The numerical simulations are performed in this study using the CFD code LeMANS. ${ }^{5,6}$ LeMANS is a three-dimensional, parallel code that solves the Navier-Stokes equations on unstructured computational grids including thermo-chemical nonequilibrium effects. In LeMANS, the flow is modeled assuming that the continuum approximation is valid. It is also assumed that the translational and rotational energy modes can be described by a single temperature, $T_{t r}$, and that the vibrational, electronic, and electron translational energy modes are described by a different temperature $T_{v e}$. The fluid conservation equations solved in LeMANS are,

$$
\frac{\partial \mathbf{Q}}{\partial t}+\nabla \cdot\left(\mathbf{F}+\mathbf{F}_{v}\right)=\mathbf{S}
$$

where, $\mathbf{Q}, \mathbf{F}$, and $\mathbf{F}_{v}$ are the vectors of conserved variables, inviscid flux, and viscous flux, respectively, and are given by,

$$
\mathbf{Q}=\left(\begin{array}{c}
\rho_{1} \\
\vdots \\
\rho_{N S} \\
\rho \mathbf{U} \\
E_{t} \\
E_{v e}
\end{array}\right), \quad \mathbf{F}=\left(\begin{array}{c}
\rho_{1} \mathbf{U} \\
\vdots \\
\rho_{N S} \mathbf{U} \\
\rho \mathbf{U} \mathbf{U}+p I \\
\left(E_{t}+p\right) \mathbf{U} \\
E_{v e} \mathbf{U}
\end{array}\right), \quad \mathbf{F}_{v}=\left(\begin{array}{c}
\mathbf{J}_{1} \\
\vdots \\
\mathbf{J}_{N S} \\
-\tau \\
-\tau \mathbf{U}+\left(\mathbf{q}_{t r}+\mathbf{q}_{v e}\right)+\sum \mathbf{J}_{s} h_{s} \\
\mathbf{q}_{v e}+\sum \mathbf{J}_{s} e_{v e, s}
\end{array}\right)
$$

In the expressions above, $\rho_{1} \cdots \rho_{N S}$ are the species densities, $\mathbf{U}$ is the bulk velocity, $E_{t}$ and $E_{v e}$ are the total and vibrational-electron-electronic energy per unit volume of mixture, $p$ is the pressure, $I$ is the identity matrix, $\tau$ is the viscous stress tensor, $\mathbf{q}_{t r}$ and $\mathbf{q}_{v e}$ are the translational-rotational and vibrational-electronelectronic heat fluxes, $\mathbf{J}_{s}$ is the diffusion flux of species $s$, and $h_{s}$ is the enthalpy of species $s$.

The source terms vector, $\mathbf{S}$, accounts for the production/consumption of species by chemical reactions and the transfer of energy to/from the vibrational-electronic-electron mode and is given by,

$$
\mathbf{S}=\left(\begin{array}{c}
\dot{w}_{1} \\
\vdots \\
\dot{w}_{N S} \\
0 \\
0 \\
\dot{w}_{v e}
\end{array}\right)
$$

The mixture transport properties are calculated using Wilke's semi-empirical mixing rule ${ }^{7}$ with species viscosities calculated using Blottner's curve fits ${ }^{8}$ and species thermal conductivities determined using Eucken's relation. ${ }^{9}$ The mass diffusion coefficient for each species is replaced by a single binary coefficient that is computed assuming a constant Lewis number of 1.4. The mass diffusion fluxes of heavy particles are modeled using a modified version of Fick's Law, ${ }^{10}$ and the diffusion flux of electrons is calculated assuming ambipolar diffusion. The viscous stresses are modeled assuming a Newtonian fluid and Stokes' hypothesis, and the heat fluxes are modeled according to Fourier's law for all temperatures.

The set of governing equations are solved using the finite-volume method applied to unstructured grids with second-order spatial accuracy. LeMANS can simulate two-dimensional and axisymmetric flows using any mixture of quadrilateral and triangular mesh cells, and three-dimensional flows using any mixture of hexahedra, tetrahedra, prisms, and pyramids. A modified Steger-Warming Flux Vector Splitting scheme ${ }^{11}$ is used to discretize the inviscid fluxes across cell faces, which is less dissipative and produces better results in boundary layers compared to the original scheme. The viscous terms are computed using cell-centered and nodal values. LeMANS is parallelized using METIS ${ }^{12}$ to partition the computational mesh, and the Message Passing Interface (MPI) to communicate the necessary information between processors.

A new boundary condition has been implemented in LeMANS to model thermionic electron emission at the surface of a hypersonic vehicle. The production rate of electrons (in $\mathrm{mol} / \mathrm{s} / \mathrm{m}^{2}$ ) at the surface due to emission and ion recombination is calculated by, 


$$
\dot{w}_{e}=\frac{J_{e}}{Q_{e} N_{A v}}+\sum_{s=i o n s} \dot{w}_{s}
$$

where $J_{e}$ is the emission current density, $Q_{e}$ is the electron charge, $N_{A}$ is the Avogadro constant, and $\dot{w}_{i}$ is the production rate of ions. The current density is calculated using Richardson's law, ${ }^{13,14}$ which relates the current from a heated material to the temperature of the material and is given by,

$$
J_{e}=A_{R} T_{w}^{2} \exp \left(\frac{-\Phi}{k_{b} T_{w}}\right)
$$

where $T_{w}$ is the surface temperature, $k_{b}$ is the Boltzmann constant, $A_{R}$ is a material-dependent constant assumed to be equal to $1.20 \times 10^{6} \mathrm{~A} / \mathrm{m}^{2} / \mathrm{K}^{2}$ in the current study, and $\Phi$ is the work function defined as the minimum energy needed to remove an electron from the material. The work function depends on the atomic configuration at the surface of the vehicle and, therefore, varies based on the material (e.g. $4 \mathrm{eV}$ for aluminum and $2 \mathrm{eV}$ for cesium ${ }^{15}$ ). Note that in the current study, electrons can only be emitted from the surface using Richardson's law and are assumed to not recombine back to the surface. The production rates of ions (and the corresponding neutrals) are calculated assuming that the surface is fully catalytic to ions and are given by, ${ }^{16}$

$$
\dot{w}_{i}=-\frac{\rho_{i}}{M_{i}} \sqrt{\frac{R_{u} T_{w}}{2 \pi M_{i}}}
$$

The gas properties at the surface of the hypersonic vehicle are calculated in LeMANS by solving the species mass balance,

$$
-\rho_{w} D_{s, w} \nabla Y_{s, w}+\dot{m} Y_{s, w}=M_{s} \dot{w}_{s}
$$

momentum balance,

$$
p_{n}+\rho_{n} v_{n}^{2}=p_{w}+\frac{\dot{m}^{2}}{\rho_{w}}
$$

and energy balance,

$$
q_{w}=\sigma_{s b} \epsilon T_{w}^{4}+\frac{J_{e}}{Q_{e}}\left(\Phi+2 k_{b} T_{w}\right)
$$

equations to obtain the species mass fractions $\left(Y_{k, w}\right)$, gas density $\left(\rho_{w}\right)$, temperature $\left(T_{w}\right)$, and gas normal velocity $\left(v_{w}\right)$. The mass blowing rate, $\dot{m}$, is defined as the product of the electron production rate and the electron mass,

$$
\dot{m}=M_{e} \dot{w}_{e}
$$

\section{Test Case Description}

The geometry of the test case considered in this study is representative of the leading edge of a hypersonic vehicle and is shown in Fig. 1. The nose radius is $1.0 \mathrm{~cm}$ (unless otherwise stated), the length of the model is $0.25 \mathrm{~m}$, and the cone angle is $5.0^{\circ}$. The material emissivity is assumed to be equal to 1.0 . The freestream conditions are presented in Table 1 and correspond to an altitude of $60 \mathrm{~km}$. A standard finite-rate chemistry model $^{17}$ for eleven species reacting air $\left(\mathrm{N}_{2}, \mathrm{O}_{2}, \mathrm{NO}, \mathrm{N}, \mathrm{O}, \mathrm{N}_{2}^{+}, \mathrm{O}_{2}^{+}, \mathrm{NO}^{+}, \mathrm{N}^{+}, \mathrm{O}^{+}\right.$, and $\left.e\right)$ is used in this study. Park's two-temperature model ${ }^{17}$ is used to account for the effect of thermal nonequilibrium on the forward and backward chemical reaction rates. Only one-half of the leading edge geometry is considered in the numerical simulations in order to reduce the computational cost. The computational grid contains approximately 20,000 cells, with 160 cells normal to the vehicle and 125 cells along the surface. A grid convergence study using the conditions without thermionic electron emission revealed that the solution is grid-independent using this mesh. 
Table 1. Freestream conditions (*unless otherwise stated).

\begin{tabular}{cccc}
\hline $\begin{array}{c}\text { Mach } \\
\text { Number }\end{array}$ & $\begin{array}{r}\text { Velocity*, } \\
\mathrm{m} / \mathrm{sec}\end{array}$ & $\begin{array}{r}\text { Density, } \\
\mathrm{kg} / \mathrm{m}^{3}\end{array}$ & $\begin{array}{c}\text { Temperature, } \\
\mathrm{K}\end{array}$ \\
\hline 19.4 & 6000 & $2.3 \times 10^{-4}$ & 238 \\
\hline
\end{tabular}

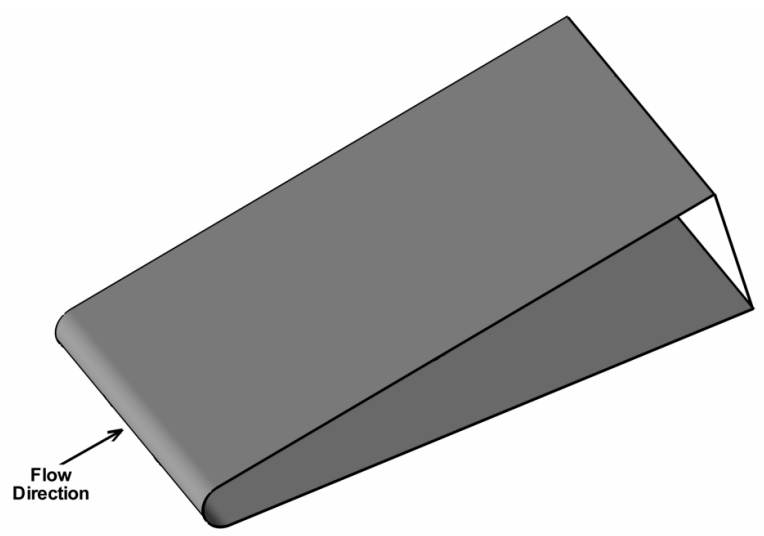

(a) 3D View

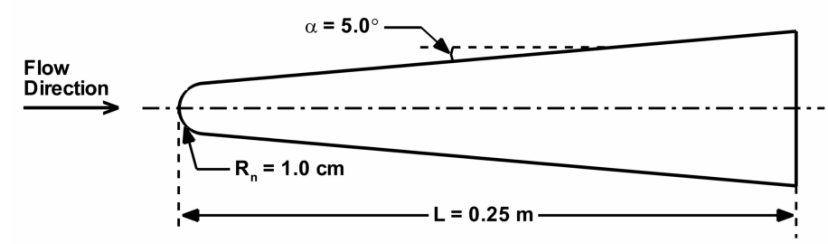

(b) Cross-Sectional View

Figure 1. Model geometry.

\section{Numerical Results}

The goal of this study is to perform a conceptual analysis of possible cooling effects caused by thermionic electron emission at the leading edges of hypersonic vehicles that employ thermo-electric TPS materials. A parametric study is carried out to understand the effects of the material work function, the freestream velocity, and the leading edge geometry on this cooling effect. Then, the effects of the electric field generated by the electron emission are approximated and discussed.

The flowfield features for the conditions investigated in this study are highlighted in Fig. 2 which shows temperature contours. The top half corresponds to the results obtained without thermionic emission, and the bottom half is for the results obtained with thermionic emission and assuming a material work function of $2.0 \mathrm{eV}$. The case without thermionic emission is analogous to using a thermo-electric material with a work function that is much greater than the surface temperature. The flow for both cases is characterized by a strong bow shock that develops around the vehicle. The fluid temperature rises to over 15,000 K across the bow shock before decreasing in the shock layer. The temperature close to the vehicle is on the order of $2500 \mathrm{~K}$, which can induce thermionic electron emission for certain thermo-electric materials. The figure also shows that thermionic emission has an overall small effect on the flowfield features.

Figure 3 presents the distribution of the temperature and number densities of electrons and ions along the stagnation line for the cases without emission (baseline) and a work function of $2.0 \mathrm{eV}$. Figure 3(a) shows overall close agreement in the calculated temperature distributions between the baseline case and the 2.0 $\mathrm{eV}$ case. However, the shock standoff distance is slightly smaller and the surface temperature is lower for the case with electron emission. As can be expected, the thermionic emission increases the electron number density at the surface as shown in Fig. 3(b). The ions number density also decreases closer to the leading edge for the case with thermionic emission due to recombination with electrons. The sharp decrease in the electron number density near the vehicle for the case with thermionic emission is anomalous and is believed to be caused by the charged species reaction rates. This is currently being investigated.

The temperature and heat transfer distributions along the vehicle surface are presented in Fig. 4 for the cases without electron emission (baseline) and a work function of $2.0 \mathrm{eV}$. Note that the distance along the leading edge is normalized by the nose radius. The surface temperature for both cases is largest near the stagnation point and decreases away from the leading edge, as can be seen in Fig. 4(a). The heat transfer to 


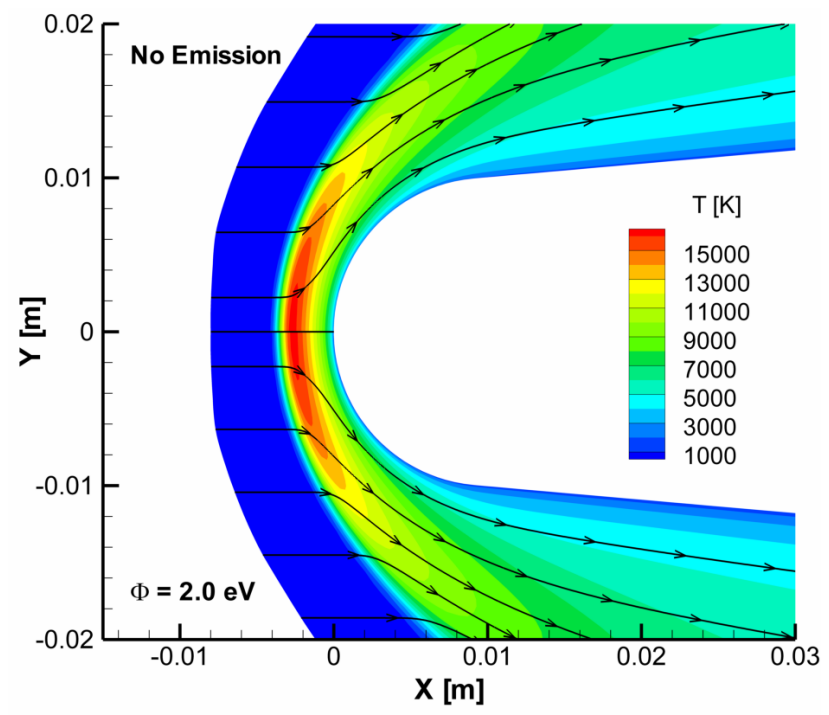

Figure 2. Temperature contours for the cases without electron emission (top) and with a material work of 2.0 eV (bottom).

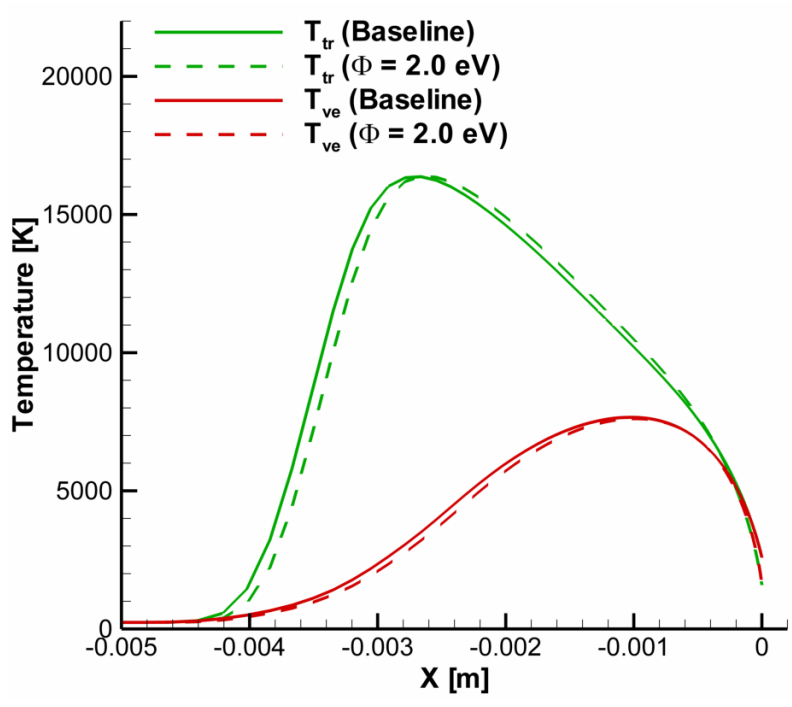

(a) Temperature

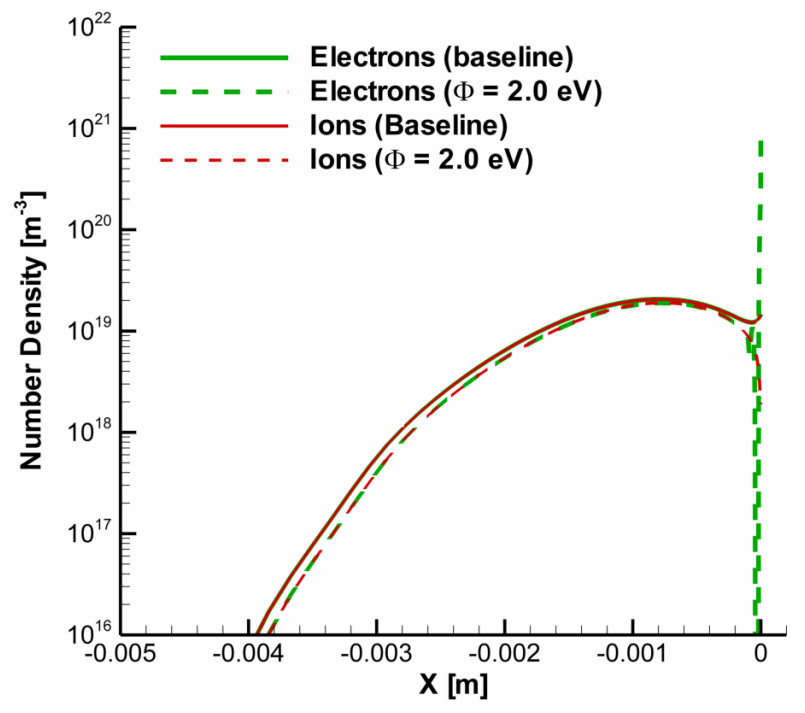

(b) Number density

Figure 3. Temperature and number density profiles along the stagnation line for the cases without electron emission (baseline) and with a material work of $2.0 \mathrm{eV}$. 
the vehicle follows a similar trend as shown in Fig. 4(b). Figure 4(a) illustrates the surface cooling effect due to electron transpiration. The temperature over the entire leading edge of the hypersonic vehicle is lower for the case with thermionic electron emission assuming a material work function of $2.0 \mathrm{eV}$ than the baseline case without emission. For instance, the temperature at the stagnation point decreases from approximately $2600 \mathrm{~K}$ for the baseline case to about $1600 \mathrm{~K}$ for the $2.0 \mathrm{eV}$ case, which corresponds to a $40 \%$ reduction. This drop in the surface temperature, however, causes an increase in the heat transfer to the vehicle as can be seen in Fig. 4(b). The reason for this is that the decrease in the surface temperature increases the temperature gradient at the wall, which according to Fourier's law, increases the heat transfer to the vehicle. The increase in the heat transfer is localized to a small region near the stagnation point, where the heat transfer is approximately $280 \mathrm{~W} / \mathrm{cm}^{2}$ for the case without thermionic emission and $320 \mathrm{~W} / \mathrm{cm}^{2}$ for the 2.0 $\mathrm{eV}$ case (corresponding to a $14 \%$ increase).

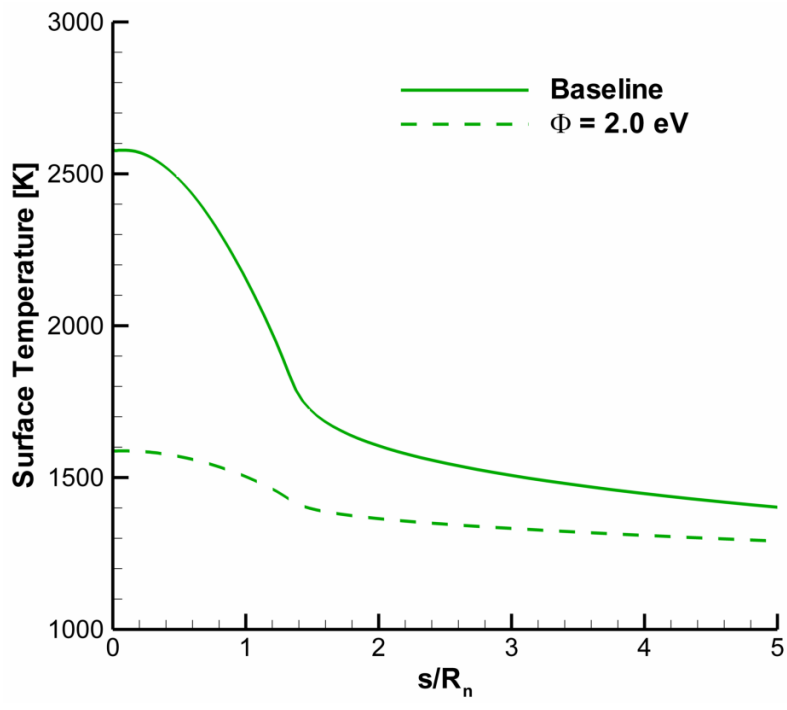

(a) Surface temperature

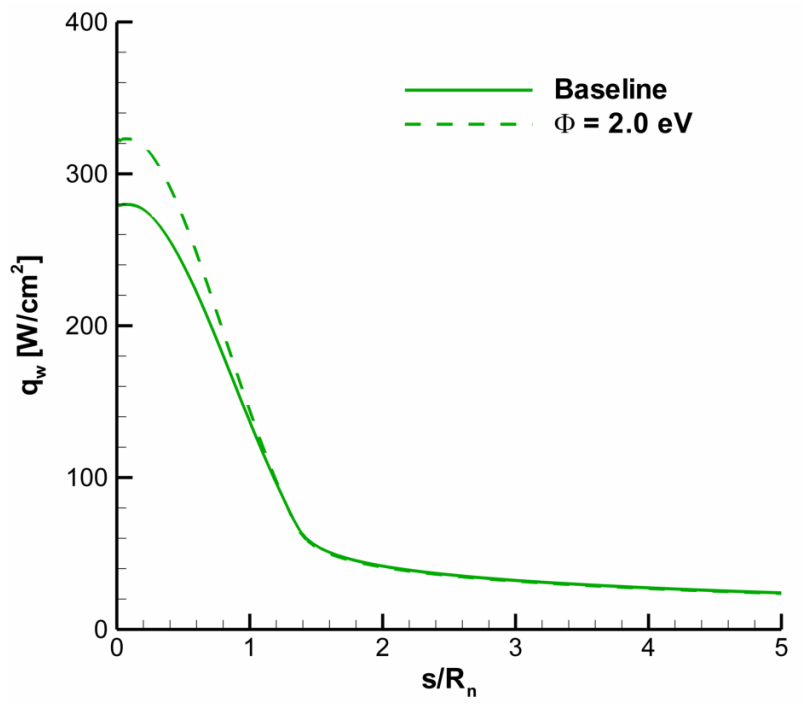

(b) Heat transfer

Figure 4. Surface temperature and heat transfer profiles for the cases without electron emission (baseline) and with a material work of $2.0 \mathrm{eV}$.

\section{A. Parametric Study}

The results presented in the previous section show that thermionic electron emission may significantly reduce the temperature along the leading edges of hypersonic vehicles. In this section, the effects of the material work function, freestream velocity, and leading edge geometry are evaluated.

\section{Material Work Function}

Two different material work functions are considered, $2.0 \mathrm{eV}$ and $2.4 \mathrm{eV}$, in order to quantify the effects of the work function on the surface properties of hypersonic vehicles that use thermo-electric TPS materials. The effects of the material work function are illustrated in Fig. 5. Notice that the surface temperature and heat transfer calculated using the two work functions are normalized by the properties obtained without electron emission in order to quantify the changes caused by the thermionic process. Figure 5(a) shows that $2.0 \mathrm{eV}$ produces a larger decrease in the surface temperature compared to $2.4 \mathrm{eV}$. At the stagnation point, the temperature decreases by approximately $40 \%$ for $2.0 \mathrm{eV}$ and $30 \%$ for $2.4 \mathrm{eV}$ relative to the baseline case without thermionic emission. Figure 5(b), however, shows that the decrease in the surface temperature is accompanied by an increase in the heat transfer to the vehicle given by Fourier's law. The figure also shows that increasing the work function decreases the rise in the heat transfer relative to the baseline case.

In order to understand why the lower work function causes a larger decrease in the surface temperature, Fig. 6 presents the distribution of the emission current density and the energy carried by the emitted electrons away from the surface computed for the $2.0 \mathrm{eV}$ and $2.4 \mathrm{eV}$ work functions. The current density is calculated in LeMANS using Richardson's law (Eqn. 6). The current density for the $2.0 \mathrm{eV}$ case is larger 


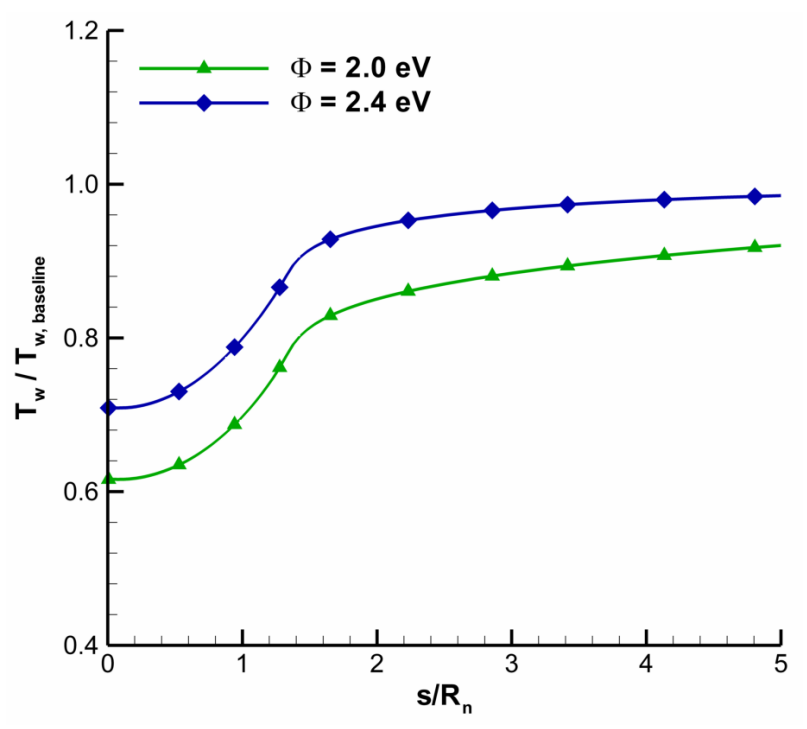

(a) Normalized surface temperature

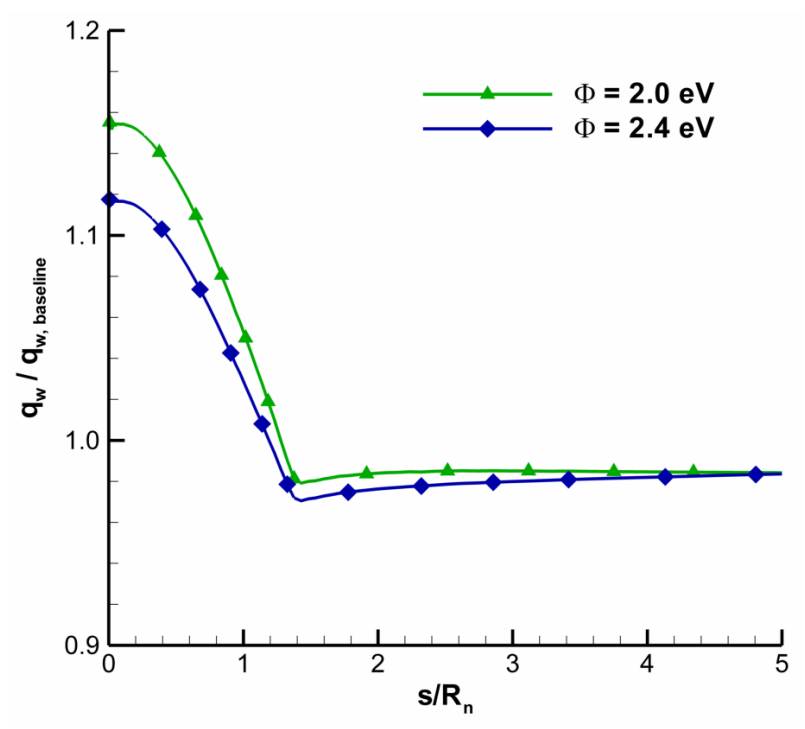

(b) Normalized heat transfer

Figure 5. Normalized surface temperature and heat transfer profiles for different material work functions.

than for the $2.4 \mathrm{eV}$ case over most of the leading edge. This suggests that more electrons are emitted at the surface which carry more energy away from the vehicle. The energy carried by the emitted electrons is shown in Fig. 6(b) and is defined as $J_{e} / Q_{e}\left(\Phi+2 k_{b} T_{w}\right)$. The lower work function increases the energy carried away from the vehicle by the emitted electrons which increases the cooling effect of the thermionic process.

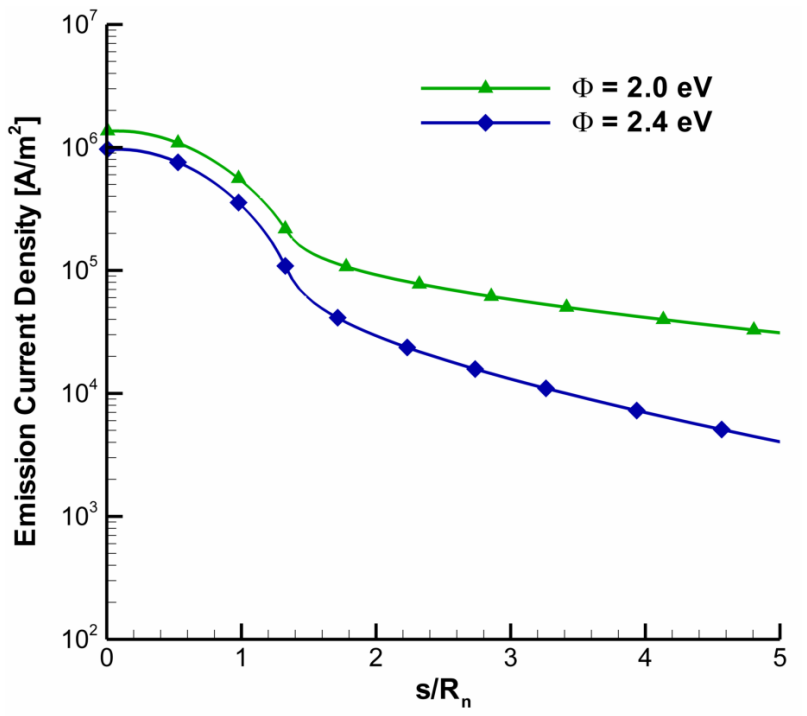

(a) Emission current density

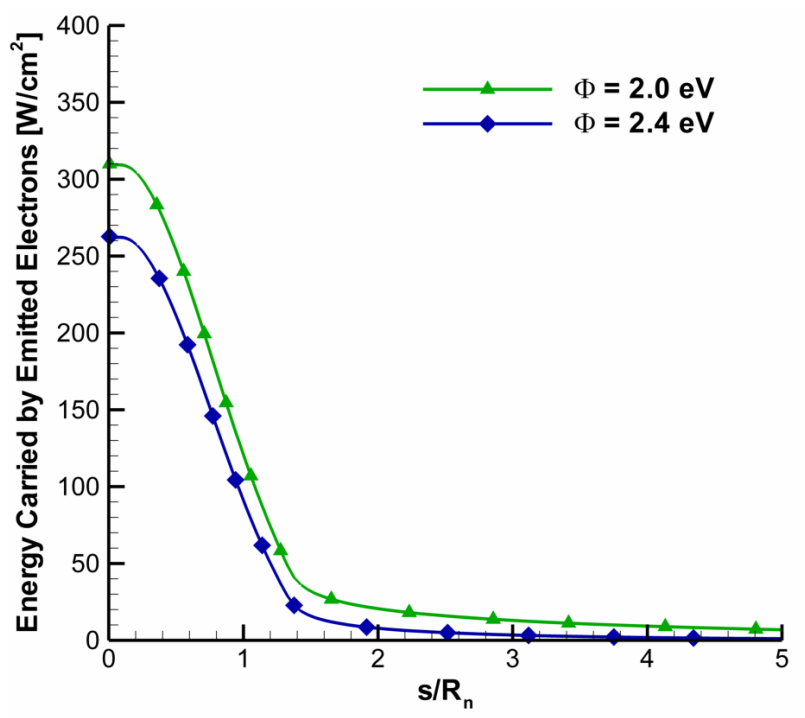

(b) Energy carried by emitted electrons

Figure 6. Emission current density and energy transported by the emitted electrons for different material work functions.

\section{Freestream Velocity}

The effects of the freestream velocity are investigated in this section by considering four different velocities: $4 \mathrm{~km} / \mathrm{s}, 6 \mathrm{~km} / \mathrm{s}, 7 \mathrm{~km} / \mathrm{s}$, and $8 \mathrm{~km} / \mathrm{s}$. The rest of the freestream conditions are equal to the values given in Table 1. Figure 7 presents temperature contours computed for freestream velocities of $4 \mathrm{~km} / \mathrm{s}$ and $8 \mathrm{~km} / \mathrm{s}$. 
The top half of the contours are calculated without thermionic emission and the bottom half are obtained assuming a work function of $2.0 \mathrm{eV}$. The figure shows that the higher freestream velocity decreases the bow shock standoff distance and increases the temperature in the shock layer. The temperature distribution calculated using a work function of $2.0 \mathrm{eV}$ is overall similar to the baseline solution without emission.

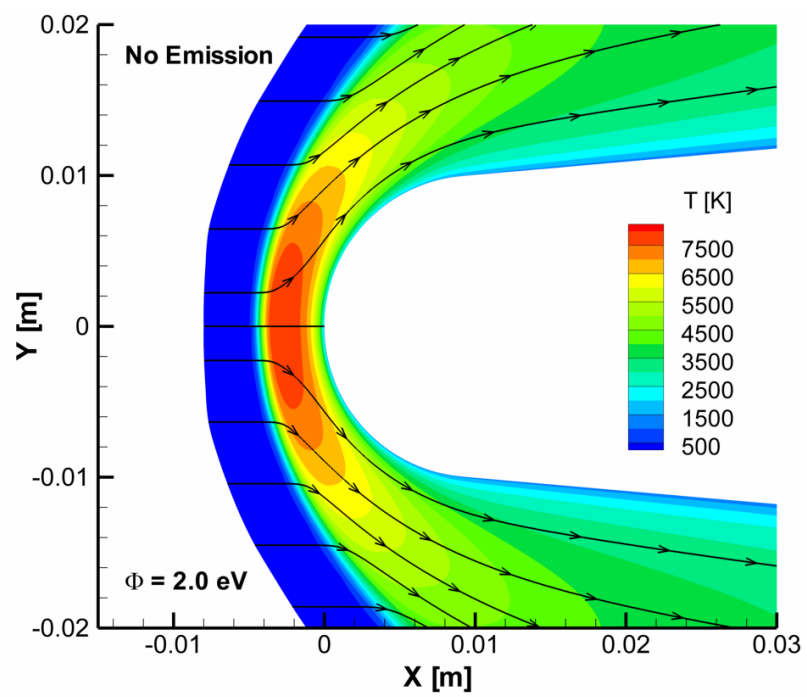

(a) $U_{\infty}=4 \mathrm{~km} / \mathrm{s}$

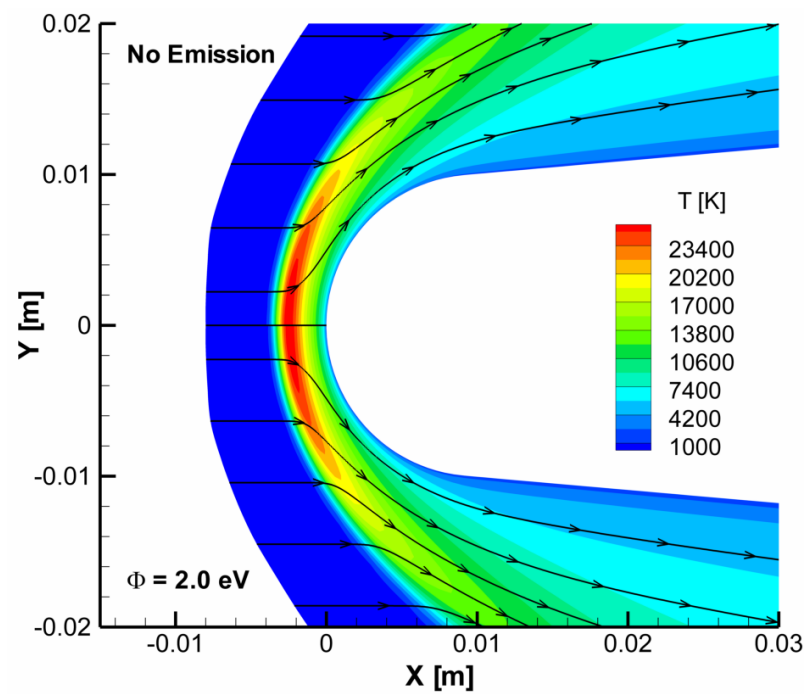

(b) $U_{\infty}=8 \mathrm{~km} / \mathrm{s}$

Figure 7. Temperature contours for the cases without electron emission (top) and with a material work of 2.0 $\mathrm{eV}$ (bottom) for different freestream velocities.

The temperature and heat transfer distributions along the surface of the vehicle calculated for the different freestream velocities without thermionic emission are presented in Fig. 8. Figure 8(a) shows that the surface temperature increases as the freestream velocity is increased. The stagnation temperature rises from approximately $2000 \mathrm{~K}$ for the $4 \mathrm{~km} / \mathrm{s}$ case to about $3100 \mathrm{~K}$ for the $8 \mathrm{~km} / \mathrm{s}$ case. Figure $8(\mathrm{~b})$ shows that the heat transfer to the vehicle also increases as the freestream velocity increases. This result can be expected based on the analytical theory of Fay-Riddell (see Eq. 1).

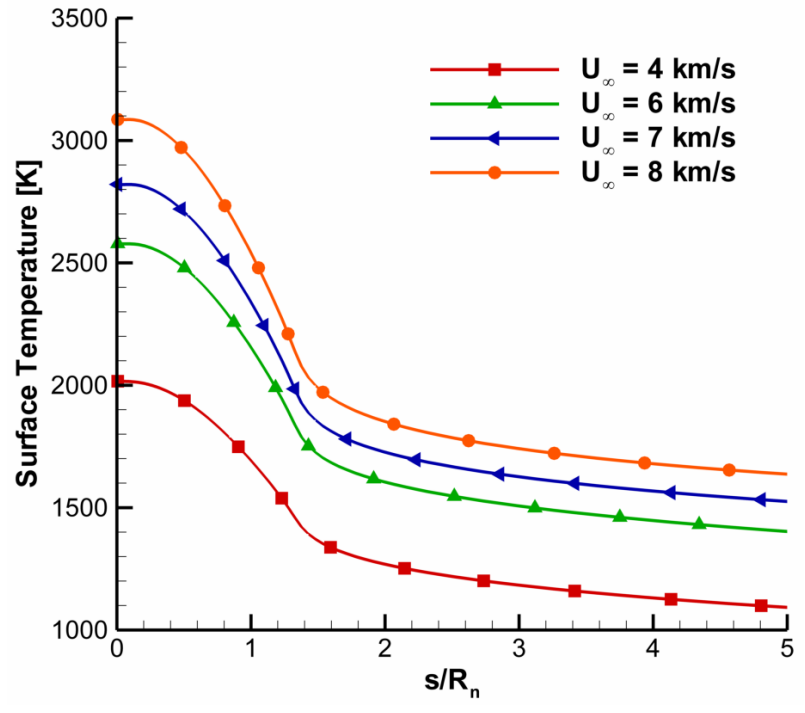

(a) Surface temperature

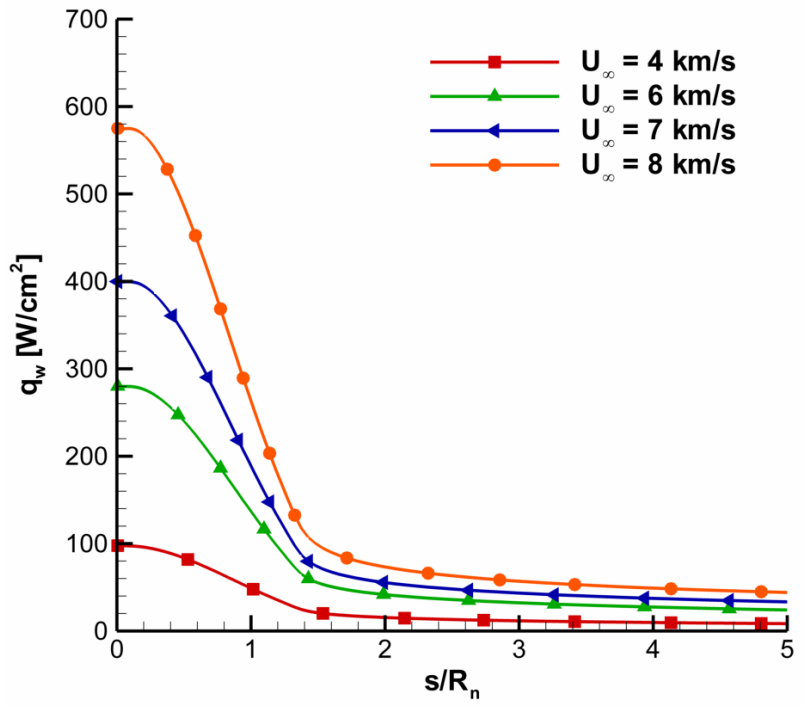

(b) Heat transfer

Figure 8. Surface temperature and heat transfer profiles for different freestream velocities without electron emission (baseline).

The normalized temperature and heat transfer distributions along the surface computed with thermionic 
emission and a material work function of $2.0 \mathrm{eV}$ at different freestream velocities are presented in Fig. 9. Thermionic emission produces a surface cooling effect for all four freestream velocities considered in this study as can be seen in Fig. 9(a). The figure also shows that the surface temperature reduction compared to the baseline case without thermionic emission increases as the freestream velocity increases. The stagnation point temperature is approximately $50 \%$ and $20 \%$ lower for a freestream velocity of $8 \mathrm{~km} / \mathrm{s}$ and $4 \mathrm{~km} / \mathrm{s}$, respectively, with thermionic electron emission. The reduction in surface temperature is again accompanied by an increase in the heat transfer relative to the baseline cases particularly in the region close to the stagnation point as shown in Fig. 9(b). This suggests that the electron transpiration cooling effect becomes more significant as the freestream velocity increases. Although not shown, these trends also extend to the solutions obtained assuming a material work function of $2.4 \mathrm{eV}$.

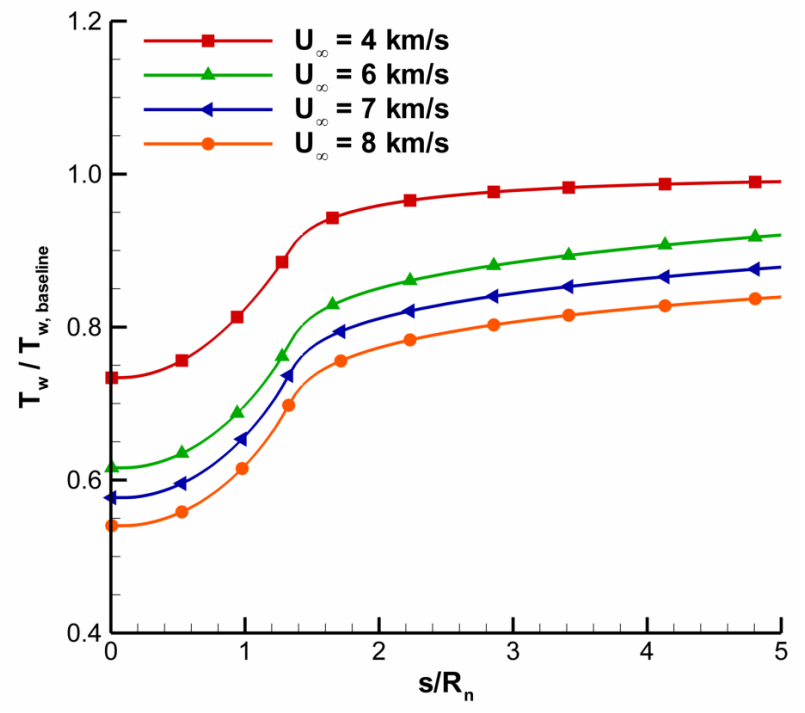

(a) Normalized surface temperature

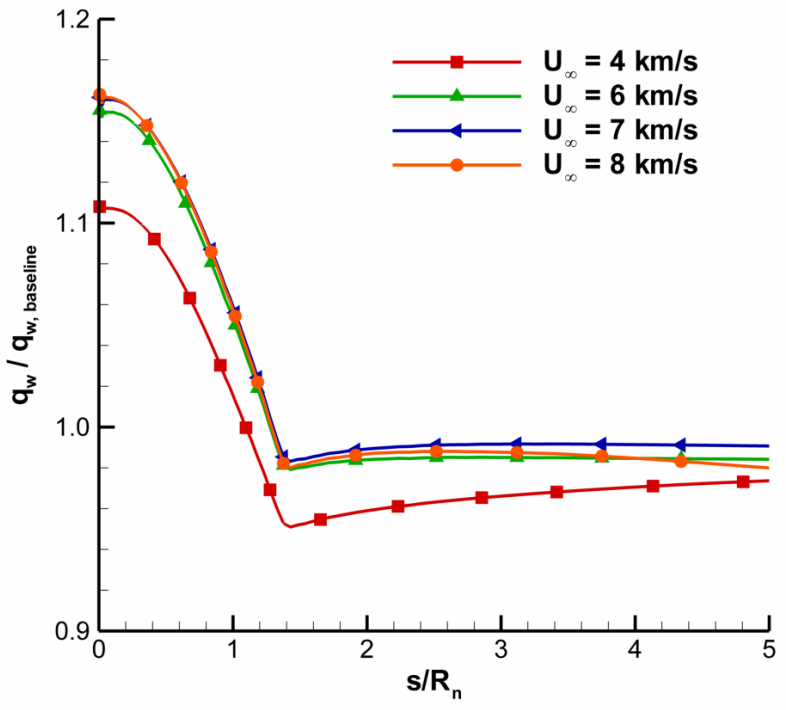

(b) Normalized heat transfer

Figure 9. Normalized surface temperature and heat transfer profiles for different freestream velocities $(\Phi=2.0$ $\mathrm{eV})$.

The increase in the electron transpiration cooling effects can once again be explained by examining the emission current density and the energy transported by the emitted electrons away from the vehicle surface shown in Fig. 10. The emission current density in Fig. 10(a) increases as the freestream velocity increases since the heat transfer to the vehicle rises (see Eq. 1). The increase in the emission current suggests that more electrons are emitted at the surface which can carry more energy away from the vehicle as shown in Fig. 10(b), which augments the cooling effect of the thermionic process.

\section{Leading Edge Geometry}

The effects of the leading edge geometry are evaluated in this section by considering two different nose radii: $1.0 \mathrm{~cm}$ and $12.7 \mathrm{~cm}$ (5 inches). The length of the vehicle is scaled up for the $12.7 \mathrm{~cm}$ while the cone-angle is the same as the $1.0 \mathrm{~cm}$ geometry (i.e. $5^{\circ}$ ). Figure 11 presents temperature contours calculated using the two different leading edge geometries with (bottom) and without (top) thermionic electron emission. The bow shock standoff distance is larger for the $12.7 \mathrm{~cm}$ geometry, while the temperature in the shock layer is greater for the $1.0 \mathrm{~cm}$ geometry. Thermionic electron emission has an overall small effect on the flowfield features even for the larger geometry.

Figure 12 presents a comparison of the surface temperature and heat transfer for the case without thermionic emission for the two different leading edge geometries. The surface temperature shown in Fig. 12 (a) is lower for the larger geometry. At the stagnation point, the temperature is approximately $2600 \mathrm{~K}$ for the $1.0 \mathrm{~cm}$ geometry and $1700 \mathrm{~K}$ for the $12.7 \mathrm{~cm}$ geometry. The heat transfer to the vehicle is much greater for the $1.0 \mathrm{~cm}$ geometry than for the $12.7 \mathrm{~cm}$ geometry as can be seen in Fig. 12(b). At the stagnation point, the heat transfer calculated for the $1.0 \mathrm{~cm}$ geometry is over a factor of five larger than for the 12.7 cm geometry. 


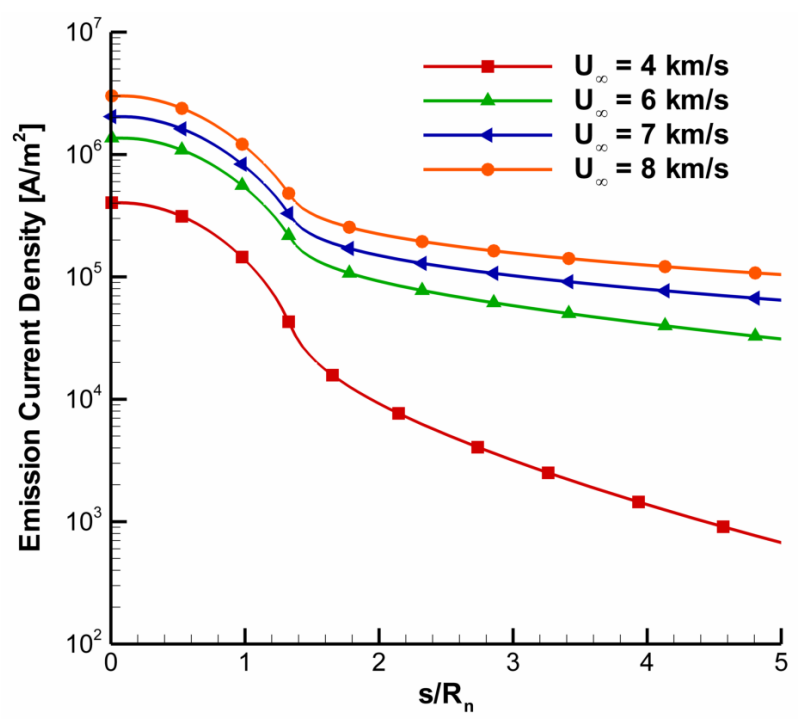

(a) Emission current density

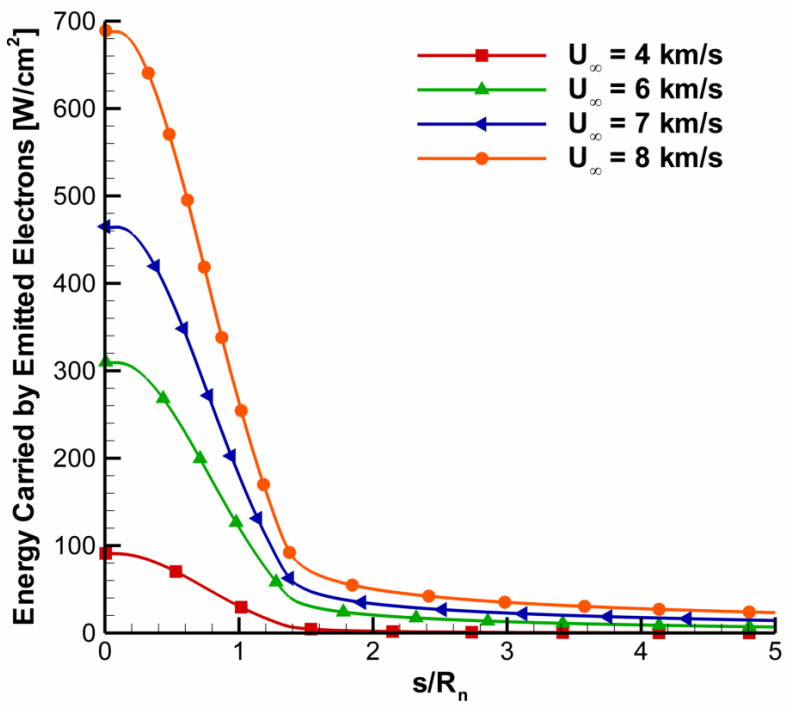

(b) Energy carried by emitted electrons

Figure 10. Emission current density and energy transported by the emitted electrons for different freestream velocities $(\Phi=2.0 \mathrm{eV})$.

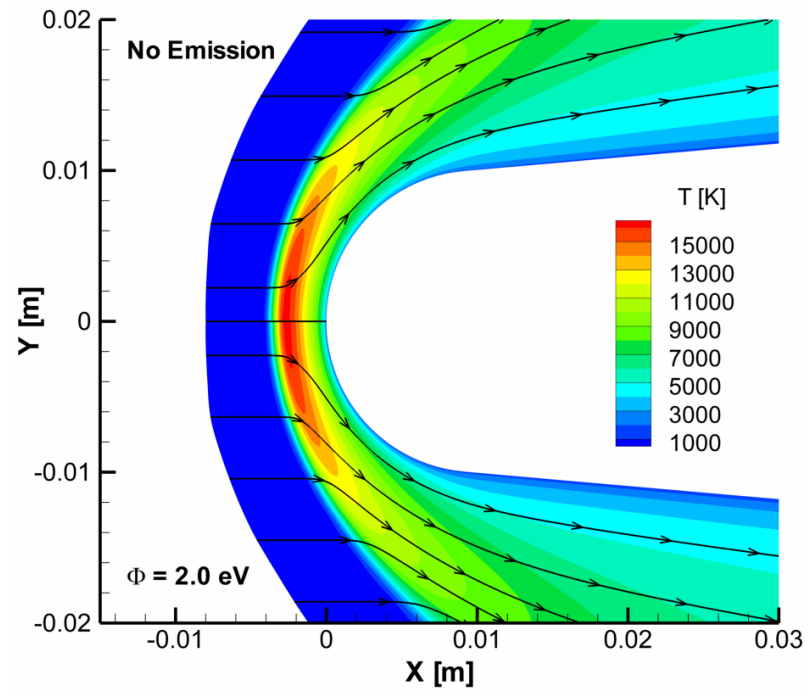

(a) $R_{n}=1.0 \mathrm{~cm}$

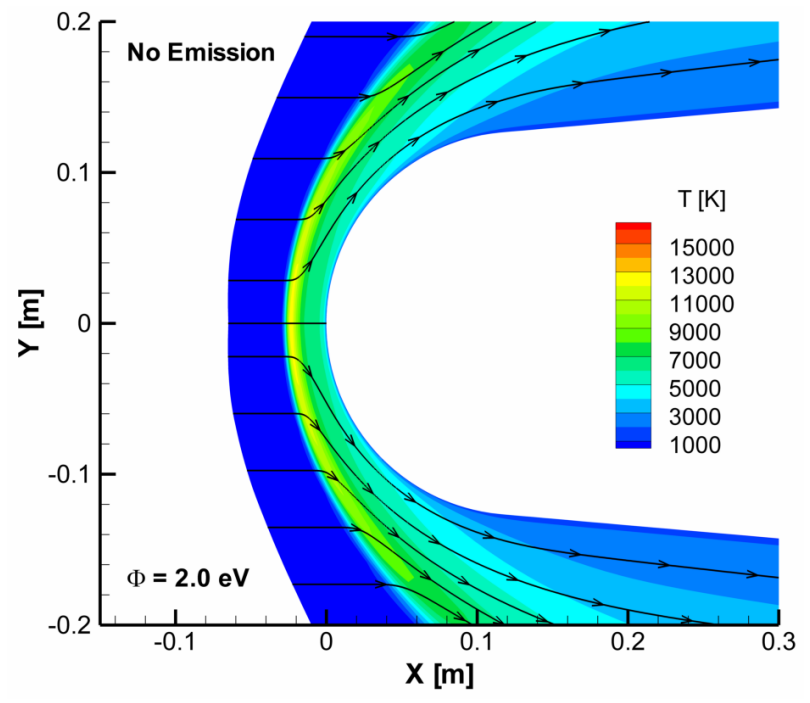

(b) $R_{n}=12.7 \mathrm{~cm}$ (note length scale)

Figure 11. Temperature contours for the cases without electron emission (top) and with a material work of $2.0 \mathrm{eV}$ (bottom) for different leading edge geometries without emission. 


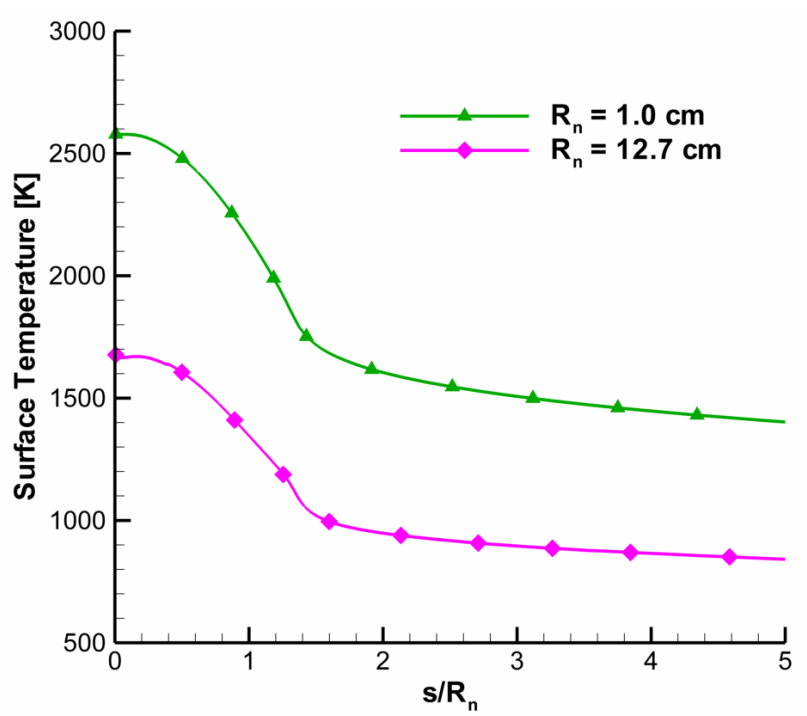

(a) Surface temperature

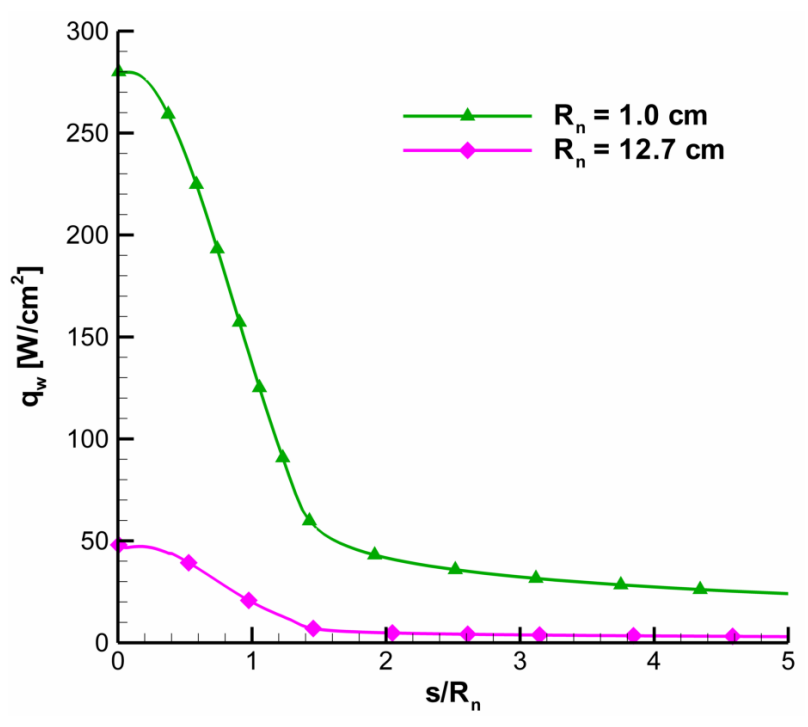

(b) Heat transfer

Figure 12. Surface temperature and heat transfer profiles for different leading edge geometries without electron emission (baseline).

The effects of the leading edge geometry on the electron transpiration cooling are once again evaluated by examining the normalized temperature and heat transfer distributions along the surface presented in Fig. 13. Figure 13(a) shows that the thermionic emission reduces the surface temperature compared to the baseline case without thermionic emission for both the $1.0 \mathrm{~cm}$ and $12.7 \mathrm{~cm}$ geometries. However, the temperature reduction due to the thermionic process is smaller for the larger geometry $(12.7 \mathrm{~cm})$ and is localized to the leading edge. The stagnation point temperature is smaller compared to the baseline case without thermionic emission by approximately $40 \%$ and $20 \%$ for the $1.0 \mathrm{~cm}$ and $12.7 \mathrm{~cm}$ geometries, respectively. The larger geometry also produces a smaller increase in the heat transfer to the vehicle when thermionic emission is modeled as can be seen in Fig. 13(b). These results indicate that the effects of thermionic electron emission become more important as the nose radius of the hypersonic vehicle decreases. Although not shown, the solutions obtained assuming a material work function of $2.4 \mathrm{eV}$ follow similar trends.

The emission current density and energy transported by the emitted electrons away from the vehicle are presented in Fig. 14 for the two geometries considered in this study. The emission current density for the $12.7 \mathrm{~cm}$ geometry is approximately an order of magnitude smaller than the current density calculated for the $1.0 \mathrm{~cm}$ geometry as can be seen in Fig. 14(a). The figure also shows that the emission current for the $12.7 \mathrm{~cm}$ geometry decreases to low values downstream of the leading edge. As a result, the energy carried by the emitted electrons away from the vehicle surface is smaller and localized to near the nose region as shown in Fig. 14(b).

\section{B. Approximation of Electric Field Effects}

The numerical results presented in the previous section are calculated by assuming that the flow is charge neutral. However, thermionic electron emission increases the number of electrons in the flow as can be seen in Fig. 3(b), which can cause a buildup of negative charge near the vehicle. This charge imbalance can produce an electric field that may influence the ionized flow around the hypersonic vehicle and change the cooling effect of the thermionic process. Electric field effects can be modeled using CFD by modifying the fluid governing equations. This section presents recent progress made in an ongoing effort to increase the physical accuracy of the numerical method used to predict the electron transpiration cooling effect for thermo-electric materials.

The fluid governing equations given in Eq. 2 can be augmented to model electric field effects by including 


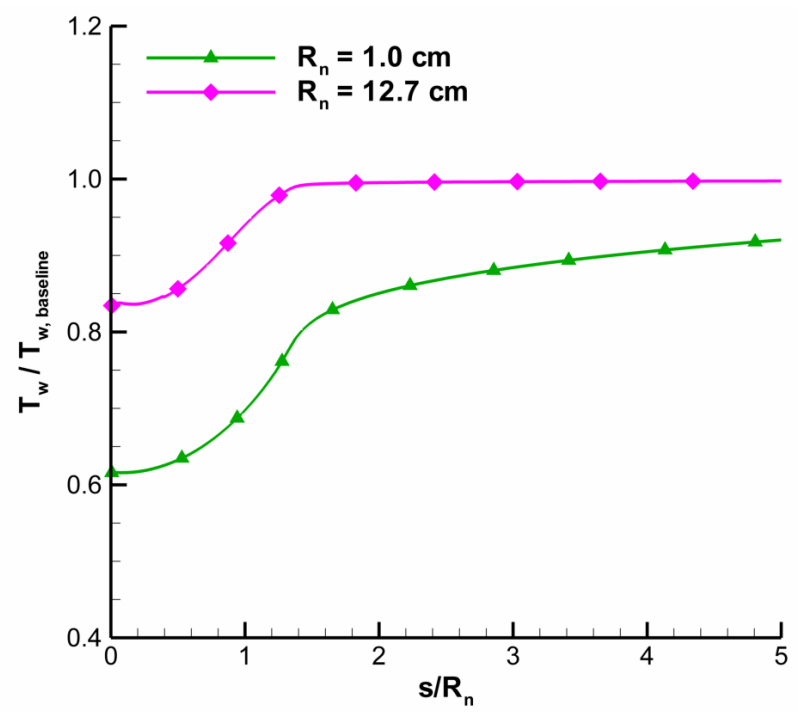

(a) Normalized surface temperature

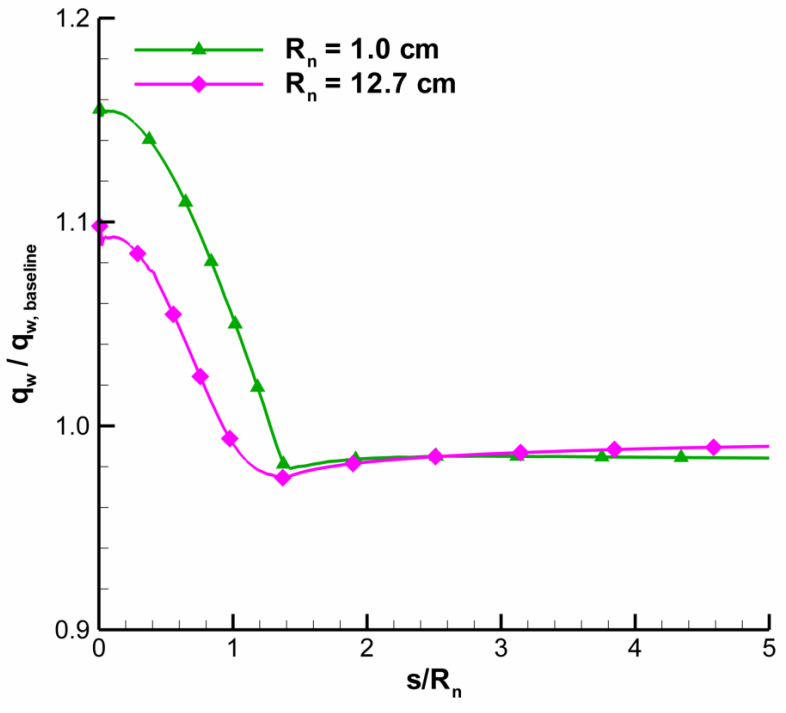

(b) Normalized heat transfer

Figure 13. Normalized surface temperature and heat transfer profiles for different leading edge geometries $(\Phi=2.0 \mathrm{eV})$.

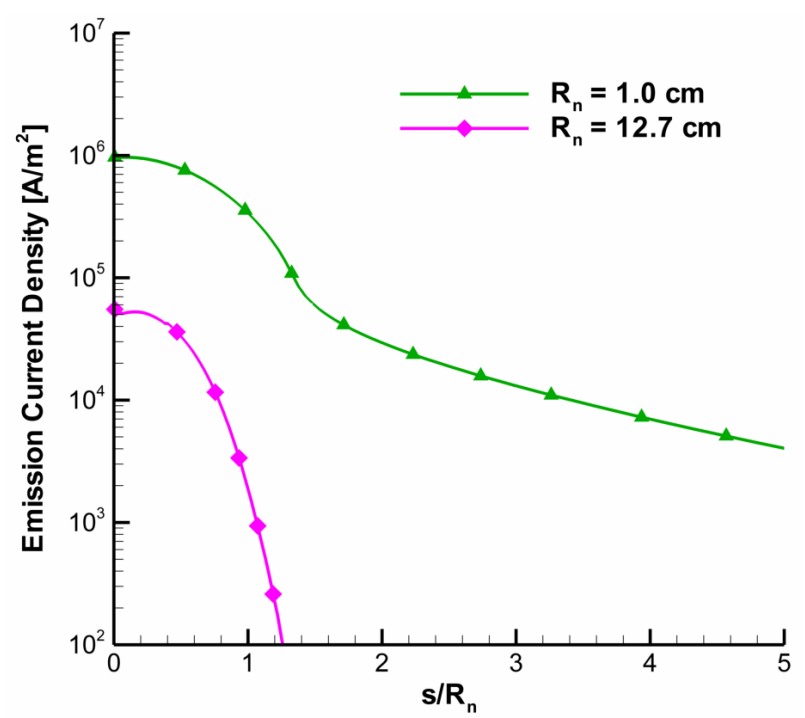

(a) Emission current density

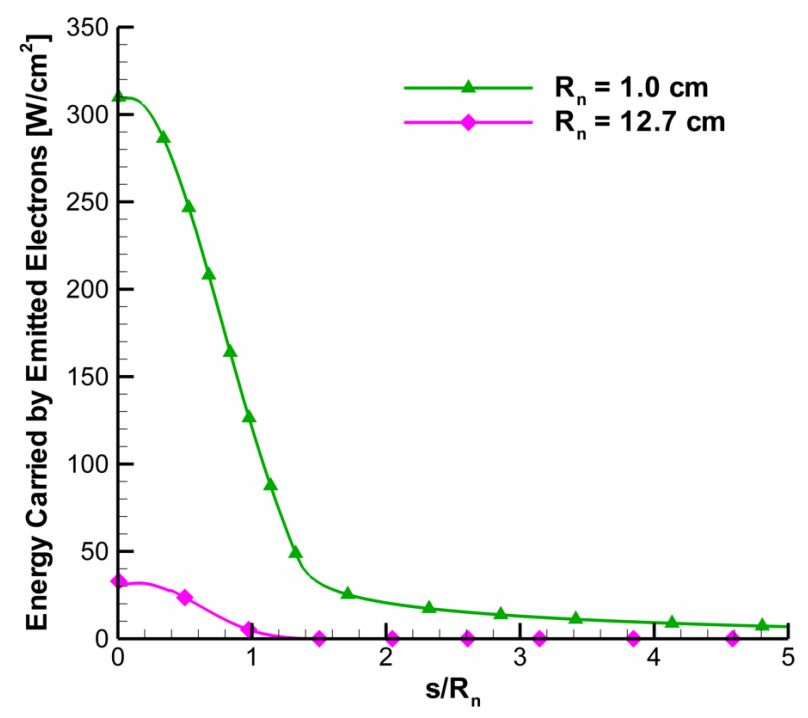

(b) Energy carried by emitted electrons

Figure 14. Emission current density and energy transported by the emitted electrons for different leading edge geometries $(\Phi=2.0 \mathrm{eV})$. 
the following source terms, ${ }^{18-20}$

$$
\mathbf{S}_{E}=\left(\begin{array}{c}
0 \\
\vdots \\
0 \\
\sum_{s} Q_{e} n_{s} C_{s} \mathbf{E} \\
\mathbf{j} \cdot \mathbf{E} \\
(\mathbf{j} \cdot \mathbf{j}) / \sigma
\end{array}\right)
$$

where, $Q_{e}$ is the elementary charge, $n_{s}$ and $C_{s}$ are the number density and charge of species $s$, respectively, $\mathbf{E}$ is the electric field, $\mathbf{j}$ is the current density, and $\sigma$ is the electric conductivity. These additional source terms account for the Lorentz force and Joule heating caused by the electric forces. The magnetic field induced by the currents within the flowfield is neglected in the current study. The accuracy of this assumption can be verified in future work by solving the Navier-Stokes equations coupled to Maxwell's equations using an algorithm similar to the one developed in Ref. 20.

The electric field can be replaced by an electric potential, $\phi$, as,

$$
\mathbf{E}=-\nabla \phi
$$

where the electric potential is calculated using Ohm's law,

$$
\mathbf{j}=\sigma \mathbf{E}
$$

and solving the steady-state current continuity equation,

$$
\nabla \cdot \mathbf{j}=\nabla \cdot(\sigma \nabla \phi)=0
$$

Equation 15 is solved at each CFD iteration using a parallel implementation of the successive overrelaxation (SOR) technique to find the distribution of the electric potential in the flowfield. A zero-gradient condition is used at the inlet, outlet, and symmetry computational boundaries. At the wall, the electric potential is calculated by assuming that the flowfield ion and electron fluxes are equal at the wall, ${ }^{21,22}$

$$
n_{i} \sqrt{\frac{k_{b}\left(T_{i}+T_{e}\right)}{m_{i}}}=\frac{1}{4} n_{e} \sqrt{\frac{8 k_{b} T_{e}}{\pi m_{e}}} \exp \left(\frac{\phi_{w}}{T_{e}}\right)
$$

where, $n_{i}$ and $n_{e}$ are the ion and electron number densities, $T_{i}$ and $T_{e}$ are ion and electron temperatures (assumed to be equal to $T_{w}$ in this study), $m_{e}$ is the electron mass, and $m_{i}$ is average mass of ions. Note that the electric potential at the wall is negative. The effect of the thermionic emission on the surface potential is not considered in the current study, but will be investigated in future work.

Several models exist to calculate the electric conductivity of the plasma. In this study, the conductivity is approximated using a semi-empirical model developed by Raizer. ${ }^{23}$ This model assumes that electronneutral collisions affect the conductivity more than the electron-ion collisions and that the ionization is in thermal equilibrium. Therefore, the conductivity is a function of temperature only as shown in Eq. 17.

$$
\sigma=8300 \times e^{-36,000 / T} \quad[\mathrm{mho} / \mathrm{m}]
$$

The electric field at the wall, computed using Eq. 13, can decrease the energy barrier that the emitted electrons must cross at the surface, which effectively reduces the work function. This is known as the Schottky effect. ${ }^{24}$ A corrected work function is calculated using Eq. 18,

$$
\Phi_{c}=\Phi-\sqrt{\frac{Q_{e}^{3} E_{w}}{4 \pi \epsilon_{0}}}
$$

where $E_{w}$ is the electric field at the wall, and $\epsilon_{0}$ is the vacuum permittivity. This corrected work function is used in Richardson's law (Eq. 6) to compute the emission current density.

The calculated electric potential and conductivity distributions in the flowfield are presented in Fig. 15 for the case without thermionic emission (top) and the case assuming a material work function equal to 2.0 
$\mathrm{eV}$ (bottom). Figure 15(a) shows that the electric potential is constant in most of the flowfield for both cases, except in a small region near the wall. This flowfield value is approximately $-1.05 \mathrm{~V}$ for the case without thermionic emission and almost $0 \mathrm{~V}$ for the case with emission. Figure 15(b) shows overall similar electric conductivity distributions in the flowfield for the two cases considered in this study. The conductivity is greatest in the post shock region and decreases close to the vehicle since the temperature decreases from a large value across the shock to a lower value at the surface (see Fig. 3(a)).

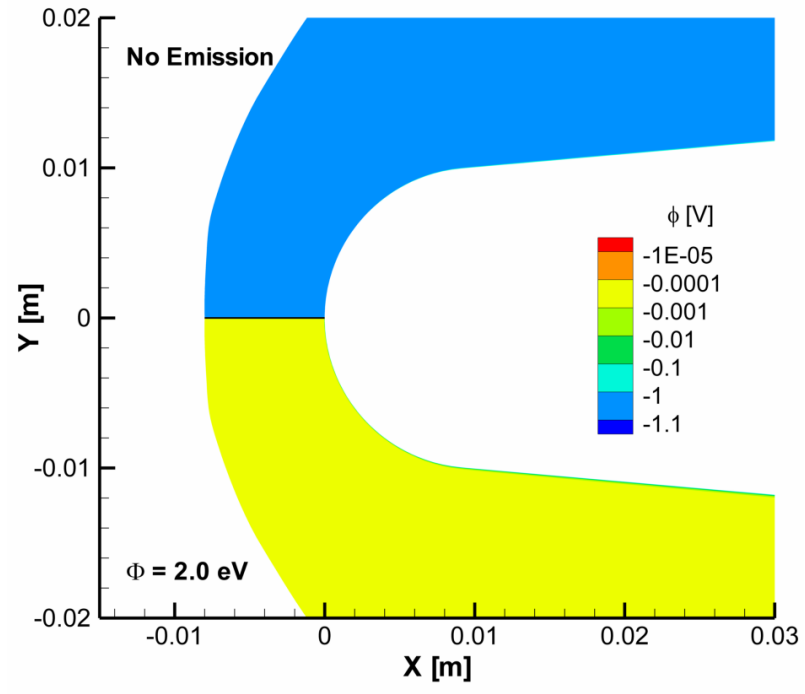

(a) Electric potential

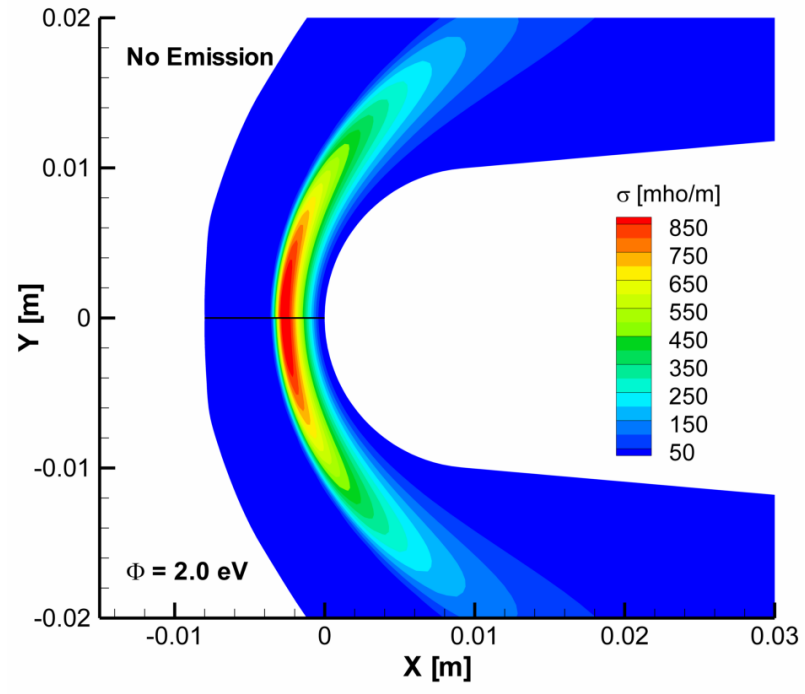

(b) Electric conductivity

Figure 15. Contours of the electric potential and conductivity for the $1 \mathrm{~cm}$ leading edge geometry at $6 \mathrm{~km} / \mathrm{s}$.

Profiles of the electric potential and conductivity along the stagnation line are shown in Fig. 16. The electric potential for the case without thermionic emission is almost constant over most of the stagnation line as can be seen in Fig. 16(a). The case with emission shows a sharp increase in the electric potential from about $-0.6 \mathrm{~V}$ at the wall to almost $0 \mathrm{~V}$ everywhere else along the stagnation line. The difference in the electric potential between the case with and without thermionic emission can be explained by examining the electric conductivity along the stagnation line shown in Fig. 16(b). The conductivity profiles obtained for the two cases are in overall close agreement, except at the wall where the conductivity calculated for the $2.0 \mathrm{eV}$ case is several orders of magnitude smaller compared to the solution computed without thermionic emission. The reason for this is that since the electric conductivity is only a function of temperature in the Raizer model, the lower surface temperature obtained for the case with thermionic electron emission decreases the conductivity at the leading edge compared to the case without thermionic emission. This lower conductivity at the surface causes the sharp increase in the electric potential observed in Fig. 16(a).

The effects of the electric field on the predicted surface properties are presented in Fig. 17 for the cases with and without thermionic electron emission. The results show that the calculated electric field has almost no effect on the baseline case without electron emission. For the case with emission, the electric field has almost no effect on the surface properties, except in a small region at the leading edge where the surface temperature slightly decreases as can be seen in Fig. 17(a). This is caused by the Schottky effect, which reduces the effective work function. These results suggest that the calculated electric field is too small to change the cooling properties of the thermionic emission for the conditions considered in this study. However, more work is needed to increase the physical accuracy of numerical method used to predict the aerothermal properties of hypersonic vehicles with thermo-electric TPS materials.

\section{Conclusions and Future Work}

The goal of the present research is to perform a conceptual analysis of potential cooling effect due to thermionic electron emission at the leading edge of hypersonic vehicles with thermo-electric TPS materials. The baseline conditions considered in this study are Mach 19.4 flow of air at an altitude of $60 \mathrm{~km}$ over 


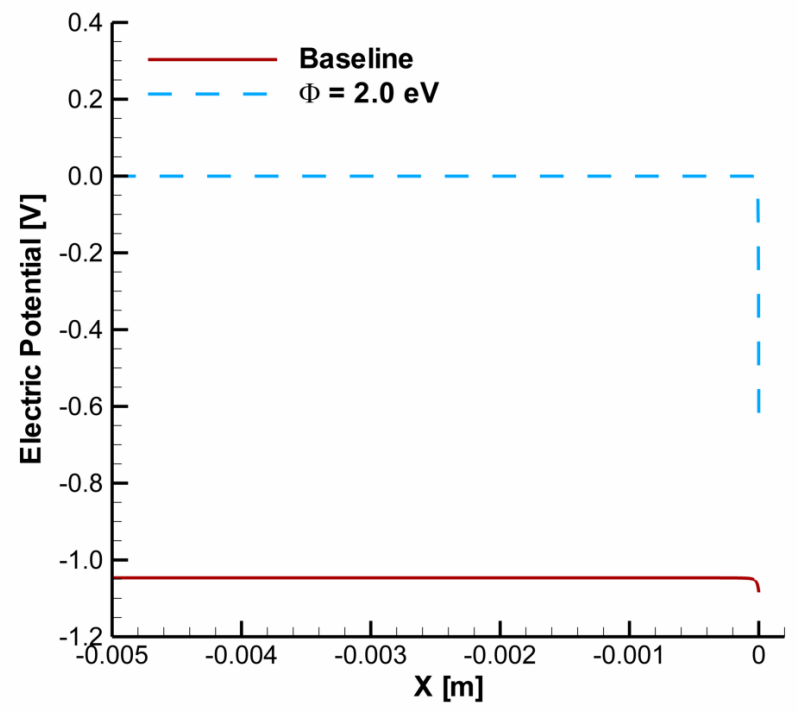

(a) Electric potential

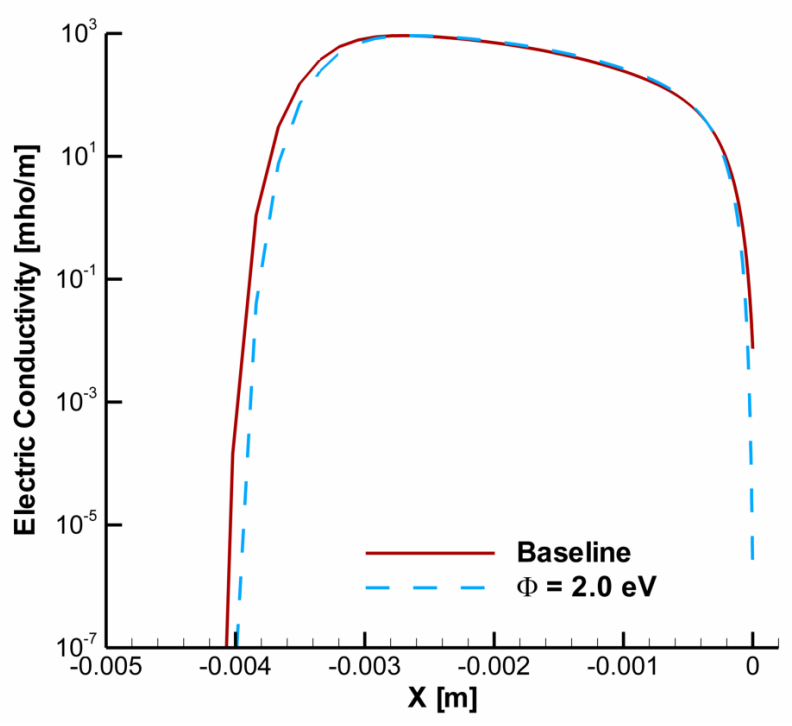

(b) Electric conductivity

Figure 16. Distribution of the electric potential and conductivity along the stagnation line for the $1 \mathrm{~cm}$ leading edge geometry at $6 \mathrm{~km} / \mathrm{s}$.

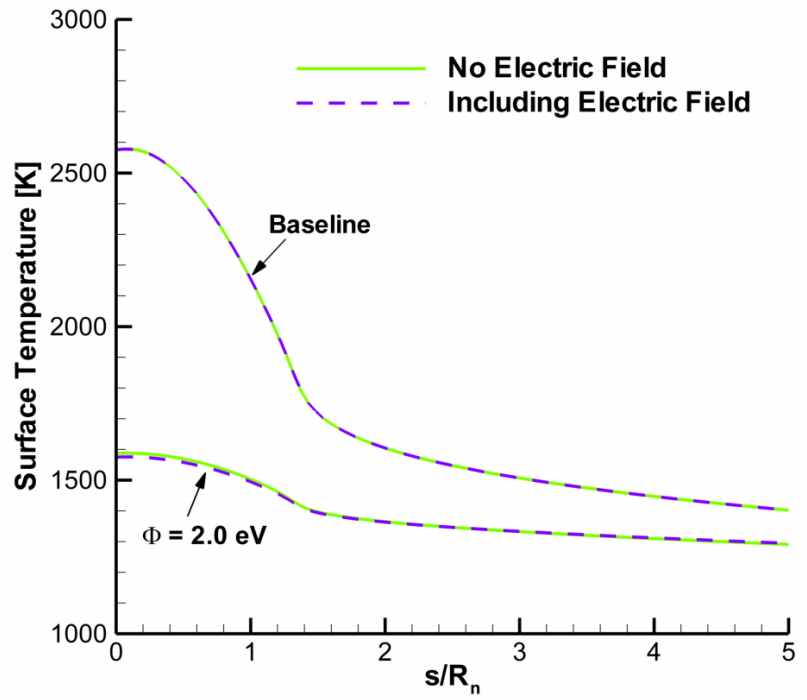

(a) Surface temperature

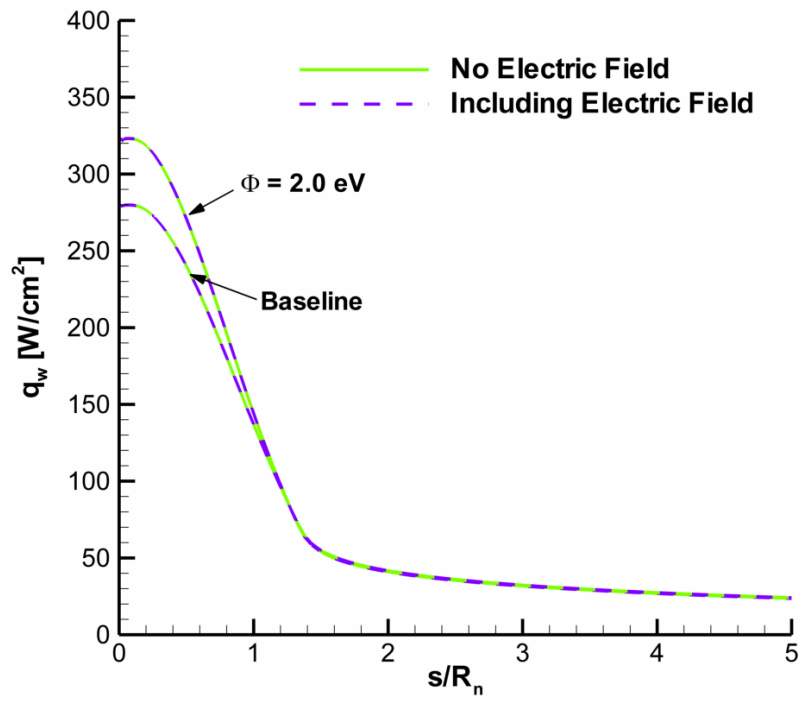

(b) Heat transfer

Figure 17. Effects of including the electric field on the surface properties for the $1 \mathrm{~cm}$ leading edge geometry at $6 \mathrm{~km} / \mathrm{s}$. 
a $1.0 \mathrm{~cm}$ cylindrical geometry that is representative of the leading edge of a hypersonic vehicle. First, the numerical approach that is used to perform this conceptual analysis was presented. Then, a parametric study was performed to understand the effects of the material work function, freestream velocity, and leading edge geometry on this potential cooling effect provided by the thermo-electric materials. The results showed that thermionic emission can reduce the surface temperature by approximately $40 \%$ assuming a material work function of $2.0 \mathrm{eV}$ for the conditions considered in this study. The results also showed that the electron transpiration cooling effect was augmented by decreasing the material work function, increasing the freestream velocity, and decreasing the leading edge radius. Some ongoing work was then described to capture the effects of the electric field on the predicted properties. The results showed that the magnitude of the calculated electric field is relatively small, which causes negligible effects on the predicted surface properties of hypersonic vehicles with thermo-electric TPS materials.

The results obtained in the current study show promising trends in the cooling properties of thermoelectric TPS materials. However, further work is needed to both continue the analysis and improve the modeling capabilities of the numerical method. For future work, the parametric study will be extended to include the effects of the freestream conditions by considering different altitudes and various angles of attack. The thermionic emission boundary condition will also be modified to allow for the recombination of emitted electrons back to the surface. Future work will also incorporate better physical models to calculate the effects of the electric field. These include: 1) account for forced diffusion (drift), 2) implement higher fidelity models for the electric conductivity, 3) estimate the effect of electron emission on the electric potential at the surface, and 4) couple the Navier-Stokes equations to Maxwell's equations to solve for both the fluid and electromagnetic properties.

\section{Acknowledgments}

The authors gratefully acknowledge support for this work from the Lockheed-Martin Corporation. In addition, we thank Dr. Luke Uribarri and Dr. Ned Allen of Lockheed for essential technical oversight. The authors also thank Dr. Erin Farbar and Mr. Kentaro Hara from the University of Michigan for several useful discussions.

\section{References}

${ }^{1}$ Walker, S. H. and Rodgers, F., "Falcon Hypersonic Technology Overview," AIAA Paper 2005-3253, 2005.

${ }^{2}$ Fay, J. A. and Riddell, F. R., "Theory of Stagnation Point Heat Transfer in Dissociated Air," Journal of the Aeronautics Sciences, Vol. 25, 1958, pp. 73-85.

${ }^{3}$ Voland, R. T., Huebner, L. D., and McClinton, C. R., "X-43A Hypersonic Vehicle Technology Development," AIAA Paper IAC-05-D2.6.01, 2005.

${ }^{4}$ Glass, D. E., "Physical Challenges and Limitations Confronting the Use of UHTCs on Hypersonic Vehicles," AIAA Paper 2011-2304, 2011.

${ }^{5}$ Scalabrin, L. C., Numerical Simulation of Weakly Ionized Hypersonic Flow over Reentry Capsules, Ph.D. thesis, University of Michigan, 2007.

${ }^{6}$ Martin, A., Scalabrin, L. C., and Boyd, I. D., "High Performance Modeling of Atmospheric Re-entry Vehicles," Journal of Physics: Conference Series, Vol. 341, No. 1, 2012, Article 012002.

${ }^{7}$ Wilke, C. R., "A Viscosity Equation for Gas Mixtures," Journal of Chemical Physics, Vol. 18 No. 4, 1950, pp. 517-519.

${ }^{8}$ Blottner, F. G., Johnson, M., and Ellis, M., "Chemically Reacting Viscous Flow Program for Multi-Component Gas Mixtures," Tech. rep., SC-RR-70-754, Sandia Laboratories, Albuquerque, New Mexico, 1971.

${ }^{9}$ Vincenti, W. G. and Kruger, C. H., Introduction to Physical Gas Dynamics, Krieger Publishing Company, 2002.

${ }^{10}$ Sutton, K. and Gnoffo, P. A., "Multi-Component Diffusion with Application to Computational Aerothermodynamics," AIAA Paper 1998-2575, June 1998.

${ }^{11}$ MacCormack, R. W. and Candler, G. V., "The Solution of the Navier-Stokes Equations using Gauss-Seidel Line Relaxation," Computers and Fluids, Vol. 17, 1989, pp. 135-150.

${ }^{12}$ Karypis, G. and Kumar, V., "METIS: A Software Package for Partitioning Unstructured Graphs, Partitioning Meshes, and Computing Fill-Reducing Orderings of Sparse Matrices," University of Minnesota, 1998.

${ }^{13}$ Richardson, O. W., The Emission of Electricity from Hot Bodies, Longmans, Green and Co., 1921.

${ }^{14}$ Angrist, S. W., Direct Energy Conversion, Allyn and Bacon, Inc., 1982.

${ }^{15} \mathrm{CRC}$ Handbook of Chemistry and Physics.

${ }^{16} \mathrm{Scott}$, C. D., "Wall Catalytic Recombination and Boundary Conditions in Nonequilibrium Hypersonic Flows - with Applications," Advances in Hypersonics - Modeling Hypersonic Flows, Vol. 2, 1992, pp. 176-250.

${ }^{17}$ Park, C., Nonequilibrium Hypersonic Aerothermodynamics, John Wiley \& Sons, 1990.

${ }^{18}$ Bisek, N. J., Boyd, I. D., and Poggie, J., "Three Dimensional Simulations of Hypersonic MHD Flow Control," AIAA Paper 2009-3731, 2009. 
${ }^{19} \mathrm{Kim}$, M. and Boyd, I. D., "Effectiveness of a Magnetohydrodynamics System for Mars Entry," Journal of Spacecraft and Rockets, Vol. 49, No. 6, 2012, pp. 1141-1149.

${ }^{20}$ MacCormack, R. W., "Solution of the Equations of Magneto-Fluid Dynamics with Chemical Reaction," AIAA Paper 2010-4636, 2010.

${ }^{21}$ Lieberman, M. A. and Lichtenberg, A. J., Principles of Plasma Discharges and Materials Processing, John Wiley \& Sons, 1994

${ }^{22}$ Benilov, M. S. and Marotta, A., "A model of the Cathode Region of Atmospheric Pressure Arcs," Journal of Physics D: Applied Physics, Vol. 28, No. 9, 1995, pp. 1869-1882.

${ }^{23}$ Raizer, Y. P., Gas Discharge Physics, Springer-Verlag, 1991.

${ }^{24}$ Fridman, A. and Kennedy, L. A., Plasma Physics and Engineering, Taylor \& Francis, 2004. 